\section{Plantas utilizadas como medicinais no município de Campos de Goytacazes - RJ.}

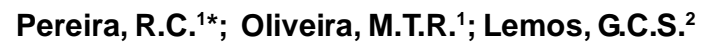

'Laboratório de Fitotecnia e Fitossanidade - Instituto Superior em Ciências Agrárias/FAETEC

${ }^{2}$ Laboratório de Fitotecnia/ Setor de Plantas Medicinais Universidade Estadual do Norte Fluminense - UENF

\section{Resumo}

Este trabalho se refere a levantamento do nível da percepção popular sobre a importância de plantas medicinais para saúde humana, tendo sido conduzido na cidade Campos dos Goytacazes, em região da Mata Atlântica, localizada no Estado do Rio de Janeiro, Brasil. Entrevistas domiciliares, conduzidas com pessoas selecionadas aleatoriamente, relacionaram 64 espécies utilizadas como medicinais, bem como os usuários obtiveram informação para seu consumo e as formas de preparo. Os resultados foram obtidos a partir de questionários semi-estruturados respondidos por residentes selecionados e estratificados segundo poder aquisitivo.

\begin{abstract}
This work reports a survey on the level of popular perception of the importance of medicinal plants on human health, and it has been conducted in the Atlantic forest town of Campos dos Goytacazes, located at Rio de Janeiro state, Brazil. Home interviews with persons selected at random related 64 species used as medicinal, as well as the source where users may obtain information on their consumption and preparation techniques. Results were obtained from semi-structured questionnaires answered by residents selected in four groups according to their income.
\end{abstract}

A Mata Atlântica é apontada como o segundo bioma tropical mais ameaçado do planeta, sendo ainda pouco estudada sob o enfoque ecológico, fitossociológico e farmacológico das espécies ali encontradas. Atualmente cerca de 80 milhões de pessoas, mais de $50 \%$ da população brasileira, vivem nessa área que, além de abrigar a maioria das cidades e regiões metropolitanas do país, sedia também os grandes pólos industriais, químicos ${ }^{1}$.

Segundo dados do $\mathrm{IBGE}^{2}$, o município de Campos dos Goytacazes, situado ao Norte do Estado Rio de Janeiro inserido na Mata Atlântica, ocupa uma área de 4.037,70 Km², uma população de 416.441 habitantes, dos quais $85 \%$ da população é urbana.

A humanidade utiliza plantas desde alguns séculos antes de Cristo, primeiro numa relação de consumidor de plantas, e mais tarde para a cura de suas enfermidades ${ }^{3,4}$.

Iniciada antes da medicina moderna, a medicina tradicional definida pela OMS como sendo a soma de todos os conhecimentos teóricos e práticos, explicáveis ou não, utilizados para diagnóstico, prevenção e tratamentos físicos, mentais ou sociais, baseados exclusivamente na experiência e observação e transmitidos verbalmente ou por escrito de geração a outra. ${ }^{5}$ Essa medicina tradicional permanece até os dias de hoje, com base em práticas mantidas há milhares de $\operatorname{anos}^{6}$, vem oferecendo uma contribuição ao desenvolvimento da ciência, a partir de conhecimentos e práticas de saúde de caráter empírico, influenciadas pelo contexto sócio-cultural, econômico e físico, no qual encontram-se inseridos?.

$\mathrm{O}$ trabalho teve como objetivo realizar levantamento do uso popular de plantas para fins medicinais pela população da cidade de Campos dos Goytacazes, RJ, coletado em quatro estratos sócio-econômicos ${ }^{8}$.

Considerando que a medicina tradicional representa um importante papel social, na medida em que, por meio de seus elementos, põe em ação os símbolos compartilhados por toda a sociedade 9 , é importante avaliar se o uso de plantas medicinais decorre de um conhecimento tradicional, embasado na experiência direta dos membros da comunidade, ou resulta de contatos com fontes externas à cultura local, como migrantes ou veículos de comunicação (livros, rádio, televisão). Situações do tipo deste último caso, indicam que o uso pode ser de introdução recente, isto é, comunidade ainda não o domina totalmente ou não está completamente familiarizada com ele, e proporcionam boa oportunidade para se estudar a difusão e a fixação de um novo conhecimento na comunidade ${ }^{10}$.

Espera-se, assim, que esse trabalho possa contribuir com o conhecimento da flora medicinal usada no município, e com informações para futuros projetos municipais na área de saúde pública.

As plantas mais utilizadas, estão apresentadas em ordem alfabética por família botânica, nome científico e nome vulgar da espécie, e forma de uso predominando em ordem decrescente chá, infusão e decocto (tabela 1).

Observou-se maior número de espécies citadas pelos entrevistados do estrato IV (30\%), seguidos pelos estratos III e II (27\%) e I (16\%) (figura 1), totalizando 64 espécies, distribuídas em 33 famílias botânicas, dentre as quais destacaram-se as famílias Lamiaceae, Asteraceae, Apiaceae, Verbenaceae e Euphorbiaceae com maior número de espécies citadas (figura 2).

Observou-se predominância da utilização de folhas (figura 3), freqüentemente a partir da combinação de plantas bem como a utilização de outros componentes no preparo, como mel e leite. A maioria dos entrevistados faz uso das plantas medicinais por indicação dos parentes e amigos, um número reduzido busca informações em livros, ou através da televisão e rádio. Mais da metade dos entrevistados recomendam o uso de alguma planta medicinal para outras pessoas. Grande parte dos entrevistados cultiva as espécies consumidas. 
Tabela 1. Plantas usadas como medicinais, forma de uso e estratos da população de Campos dos Goytacazes onde foram mencionadas.

\begin{tabular}{|c|c|c|c|c|}
\hline Família & Nome vulgar & Nome científico & $\begin{array}{l}\text { Forma de uso/ parte } \\
\text { da planta utilizada }\end{array}$ & Extrato* \\
\hline Amaranthaceae & Terramicina (anador) & Alternanthera dentata (Moech) Stuchlik & Chá (folha) & I \\
\hline Anacardiaceae & Aroeira & Schinus terebinthifolius Raddi & Tintura (folha) & III \\
\hline Anacardiaceae & Manga & Mangífera indica $\mathrm{L}$. & Xarope (folha) & IV \\
\hline Annonaceae & Graviola & Annona muricata $\mathrm{L}$ & Chá (fruto e folha) & III, IV \\
\hline Apiaceae & Erva-doce & Foeniculum vulgare Mill & Chá (folha, flor) & I, II, III \\
\hline Apiaceae & Salsinha & Hydrocotyle bonariensis Lam & Chá (folha) & II \\
\hline Apocynaceae & Pau-pereira & Aspidosperma parvifolium A.DC. & Chá (casca) & IV \\
\hline Araceae & Costela-de-adão & Philodendron pertusum & Tintura (folha) & III \\
\hline Asteraceae & Arnica & Solidago chilensis Meyen & Banho (folha) & II, III, IV \\
\hline Asteraceae & Assa-peixe & Vernonia polyanthes Lees & Chá, xarope (folha, flor) & IV \\
\hline Asteraceae & Camomila & Chamomilla recutita (L.) Rauschert & Chá (flor) & I \\
\hline Asteraceae & Carqueja & Baccharis trimera (Less.) DC. & Chá (haste, folha) & II \\
\hline Asteraceae & Falsa-camomila & Coreopsis grandiflora L. & Chá (flor) & II, III \\
\hline Asteraceae & Guaco & Mikania glomerata Spreng. & Chá (folha) & III, IV \\
\hline Asteraceae & Picão & Bidens pilosa $\mathrm{L}$. & Chá (folha) & IV \\
\hline Bignoniaceae & Carajuru & Arrabidaea chica (Bonpl.) B. verl. & Chá (folha) & I \\
\hline Bignoniaceae & Carobinha & $\begin{array}{l}\text { Tabebuia aurea (Silva Manso) } \\
\text { Benth \& Hook.f. ex S. Moore }\end{array}$ & Banho (folha) & III \\
\hline Bignoniaceae & Unha-de-gato & Macfadyena unguis-cati (L) A. H. Gentry & Chá (folha) & III \\
\hline Caesalpiniaceae & Pata-de-vaca & Bauhinia sp. & Chá (folha) & II \\
\hline Caesalpiniaceae & Sene & Senna $\mathrm{sp}$. & Chá (folha) & I, II \\
\hline Caricaceae & Mamão & Carica papaya $\mathrm{L}$. & Xarope (flor) & II, IV \\
\hline Chenopodiaceae & Mentrusto & Chenopodium ambrosioides $\mathrm{L}$. & Suco (folha) & III \\
\hline Costaceae & Cana-do-brejo & Costus spicatus (Jacq.) Sw. & Chá (planta inteira) & II \\
\hline Crassulaceae & Saião & Kalanchoe brasiliensis Camb. & Chá, xarope (folha) & II, III, IV \\
\hline Curcubitaceae & Melão-de-são-caetano & Mamordica charantia $\mathrm{L}$. & Chá (folha) & II \\
\hline Euphorbiaceae & Mamona-branca & Ricinus communis L. & Banho-de-acento (haste, folha) & IV \\
\hline Euphorbiaceae & Quebra-pedra & Phyllanthus niruri L. & Chá (planta inteira) & II \\
\hline Lamiaceae & Alfavaca & Ocimum basilicum $\mathrm{L}$. & Chá (folha) & I, II \\
\hline Lamiaceae & Alfavacão & Ocimum gratissimum $\mathrm{L}$. & Xarope (folha) & IV \\
\hline Lamiaceae & Boldo & Plectranthus barbatus Andrews & Chá (folha) & I, II, III, IV \\
\hline Lamiaceae & Hortelã & Mentha $x$ villosa Huds & Chá (folha) & I, II, III, IV \\
\hline Lamiaceae & Levante & Mentha sp. & Xarope (folha) & IV \\
\hline Lamiaceae & Poejo & Mentha pulegium $\mathrm{L}$. & Chá, xarope (folha) & II, IV \\
\hline Lamiaceae & Manjericão & Ocimum selloi Benth & Chá (folha) & I \\
\hline Lauraceae & Abacate & Persea americana Mill. & Chá (folha) & II \\
\hline Lauraceae & Canela & Cinnamomum zeylanicum Breyn & Xarope (folha) & III \\
\hline Lauraceae & Louro & Laurus nobilis L. & Chá (folha) & III \\
\hline Liliaceae & Alho & Allium sativum L. & Xarope (bulbo) & IV \\
\hline Liliaceae & Babosa & Aloe spp. & Xarope (folha) & I, III \\
\hline Liliaceae & Cebolinha & Allium fistulosum L. & Chá (folha) & II \\
\hline Malpighiaceae & Acerola & Malphighia glabra L. & Chá (folha) & II \\
\hline Myrtaceae & Goiaba & Psidium guajava L. & Chá (folha, broto) & II \\
\hline Myrtaceae & Jamelão & Syzygium cumini (L.) Skeels & Chá (folha) & IV \\
\hline Myrtaceae & Pitanga & Eugenia uniflora L. & Banho (brotos) & III \\
\hline Oxalidaceae & Carambola & Averrhoa carambola $\mathrm{L}$. & Suco (fruto) & IV \\
\hline Oxalidaceae & Limão-de-salada & Não identificada & Xarope (fruto) & IV \\
\hline Palmae & Coco & Cocos nucifera $\mathrm{L}$. & Chá (folha) & II \\
\hline Passifloraceae & Maracujá & Passiflora edulis Sims & Pó (casca); suco (fruto) & III, IV \\
\hline Piperaceae & Kava-kava & Piper methysticum Forster & Chá (??) & III \\
\hline Plantaginaceae & Tanchagem & Plantago major $\mathrm{L}$. & Chá (folha) & I, IV \\
\hline Poaceae & Capim-limão & Cymbopogon citratus (DC) Stapf. & Chá (folha) & I, II, III, IV \\
\hline Punicaceae & Romã & Punica granatum L. & Chá (casca do fruto) & IV \\
\hline Rosaceae & Maçã & Malus domestica L. & Chá (casca do fruto) & I, III \\
\hline Rosaceae & Rosa-branca & Rosa sp. & Chá (flor) & IV \\
\hline Rutaceae & Laranja & Citrus sp. L. & Chá (folha) xarope (folha, flor) & II, IV \\
\hline Rutaceae & Limão & Citrus limon (L.) Burm. F. & Xarope (fruto, folha) & I, II, IV \\
\hline Sapotaceae & Abiu roxo & Pouteria sp. & Chá (folha) & II \\
\hline Solanaceae & Berinjela & Solanum melongena & Chá (fruto) & IV \\
\hline Solanaceae & Tomate & Licopersicon esculentum Mill & Maceração (fruto) & IV \\
\hline Solanaceae & Trombeta & Datura stramonium L. & Maceração (folha) & III \\
\hline Theaceae & Chá-preto & Camellia sinensis (L.) Kuntze & Chá (folha) & I \\
\hline Verbenaceae & Erva-cidreira & Lippia alba (Mill.) N.E. Br. & Chá (folha) & I, II, III, IV \\
\hline Verbenaceae & Gervão-roxo & Stachytarpheta cayennensis (Rich) Vahl & Chá (folha) & IV \\
\hline Zingiberaceae & Cordomão & Alpinia sp. & Chá (folha) & II \\
\hline
\end{tabular}

*Estrato I- poder aquisitivo alto; estrato II- poder aquisitivo médio; estrato III- poder aquisitivo baixo e estrato IV- periferia. 


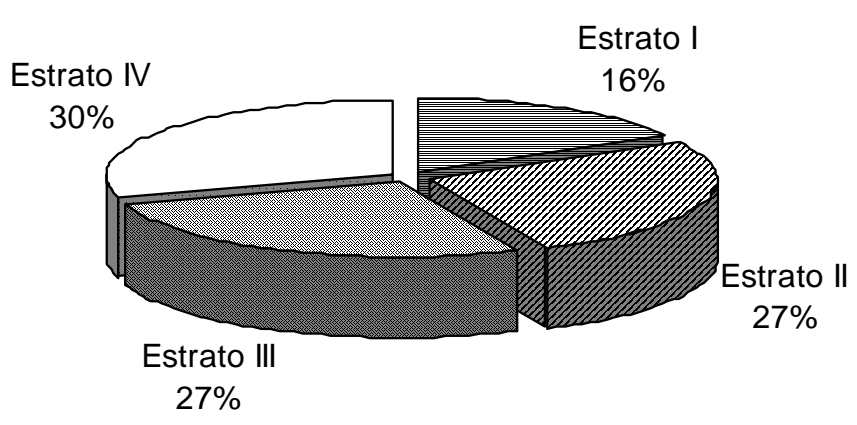

Figura 1. Porcentagem de espécies citadas em cada estrato estudado: Estrato I- população com maior poder aquisitivo; estrato II- médio poder aquisitivo; estrato III- baixo poder aquisitivo e estrato IV- população periférica.

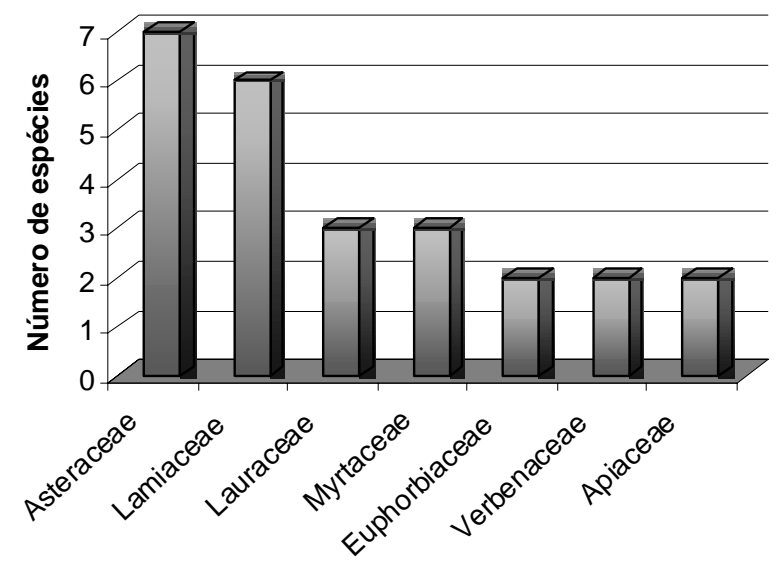

Figura 2. Famílias de plantas medicinais mais citadas no município de Campos dos Goytacazes- Rio de Janeiro.

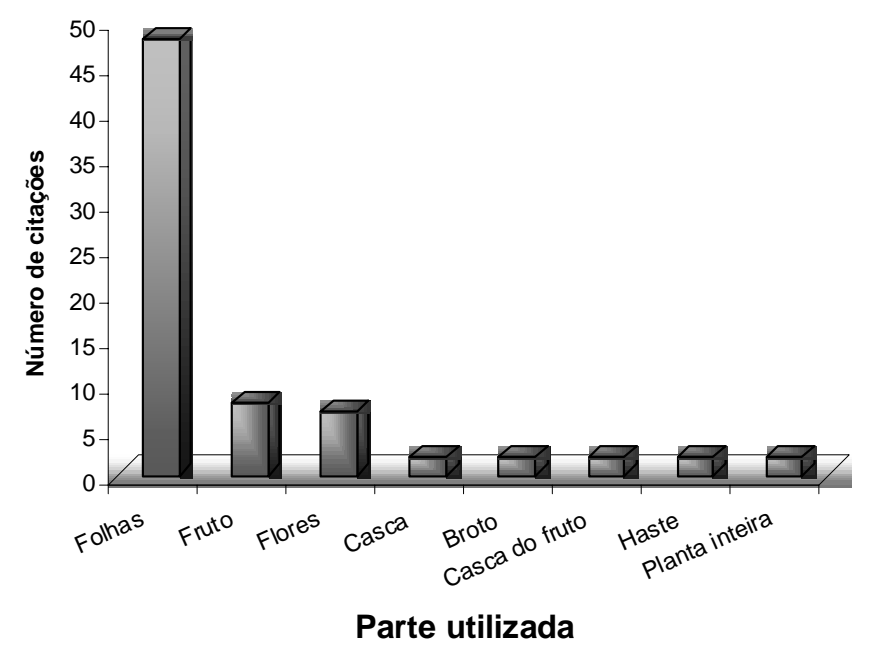

Figura 3 Partes das plantas medicinais mais utilizadas no município de Campos dos Goytacazes - Rio de Janeiro.

\section{Materiais e Métodos}

A avaliação foi feita por meio de entrevistas semi- estruturadas, com perguntas abertas sobre plantas usadas para curar ou prevenir doenças, formas de preparo (chá, ungüento, emplasto, infusão, decocto e pomada), parte da planta utilizada e listagem livre das plantas usadas pelos entrevistados ${ }^{11}$. Durante a pesquisa foram visitados quintais ou jardins das residências, procedendo-se o levantamento do material vegetal, formas de obtenção e propagação, hábito de crescimento (herbáceo, arbóreo, arbustivo, rasteiro e trepador) e sua coleta para identificação botânica quando possível. As espécies foram identificadas com o auxílio de literatura, seguindo CRONQUIST ${ }^{12}$ e por especialista.

Os questionários foram aplicados a residentes de bairros localizados em quatro estratos sócio-econômicos da cidade de Campos dos Goytacazes: estrato I- poder aquisitivo alto; estrato II- poder aquisitivo médio; estrato III- poder aquisitivo baixo e, estrato IV- periferia, num total de 48 domićlíios ${ }^{2}$.

\section{Referências}

${ }^{1}$ IUCN. Cuidando do Planeta Terra, uma estratégia para o futuro da vida. IUCN/ PNUMA / WWF. 246p. 1991.

${ }^{2}$ Instituto Brasileiro de Geografia e Estatística. Dados do censo demográfico de 2001. Disponível em http:// www.ibge.br/cidadessat/default.php.

${ }^{3}$ Marodin, S. M. e Baptista, L. R. M. Plantas utilizadas como medicinais no município de Dom Pedro de Alcântara, Rio Grande do Sul, Brasil. 1. Origem e aspectos ecológicos. Iheringia, Série Botânica, Porto Alegre, n. 56, p. 131-146, 2001.

${ }^{4}$ Neves, M. C. M. Plantas medicinais: diagnóstico e gestão. Brasília. Editora Ibama, 2001 (Série Meio Ambiente em Debate $\mathrm{n}^{\circ}$ 35). 52p., 2001.

${ }^{5}$ WHO.Traditional medicine and modern health care-Progress report by Director- General. Forty fourth world health assembly.22 March, 1991.

${ }^{6}$ Akerele, O. Medicinal plants and primary health care: an agend for action. Fitoterapia, Milano v.59, n.5, p.355-363. 1988.

${ }^{7}$ Camargo, M. T. L. A medicina popular. Rio de Janeiro: Campanha de Defesa do Folclore Brasileiro, 46p., 1976.

${ }^{8}$ Instituto Brasileiro de Geografia e Estatística. Contagem da população de 2001. Rio de Janeiro, v.2. Resultados relativos à população e os domicílios, 2003.

${ }^{9}$ Queiroz, M. S. O paradigma mecanicista da medicina tradicional ocidental moderna: uma perspectiva antropológica. Revista de Saúde Pública, v.20, n.4, p.309$317,1986$.

${ }^{10}$ Amorozo, M. C. M. A abordagem etnobotânica na pesquisa de plantas medicinais In: Stasi, L. C. di (org). Plantas medicinais: Arte e ciência: um guia interdisciplinar, São Paulo: Editora da UNESP, p. 47-68, 1996.

${ }^{11}$ Cotton, C.M. Ethnobotany: principles and applications. New York, John Wiley \& Sons, 424p, 1996.

${ }^{12}$ Cronquist, A. An Integrated system of classification floweing plants. New York: Columbia University, cap. 4, p. 326-369. 1981. 
*Autora para correspondência

Prof ${ }^{a}$. Rozimar de Campos Pereira

Rua Alvarenga Filho, 21/104

Centro, Campos dos Goytacazes, RJ

CEP 28035-125

e-mail: rozimarcp@uol.com.br 